\title{
Grand challenges and opportunities in deciphering the role of non-coding RNAs in human diseases
}

\author{
William C. S. Cho* \\ Department of Clinical Oncology, Queen Elizabeth Hospital, Kowloon, Hong Kong \\ *Correspondence: chocs@ha.org.hk
}

Non-coding RNAs (ncRNAs) were at first thought to be a non-specific noise. The vast majority of them have yet to be characterized thoroughly, but many of these ncRNAs are unlikely to represent transcriptional noise as a significant number have been shown to exhibit cell type-specific expression, localization to subcellular compartments, and association with human diseases (Wilusz et al., 2009). Although ncRNAs do not encode protein, this does not mean that such RNAs do not contain information nor have function. Widespread transcription of ncRNAs is now known to occur in all species and they are likely to represent a fundamental process in biological systems.

Most of the newly identified ncRNAs' functions are unknown, but these transcripts are increasingly recognized as regulators of gene expression. They appear to comprise a hidden layer of internal signals that control various levels of gene expression in physiology and development, including chromatin architecture/epigenetic memory (a process by which changes in gene expression are passed on via mitosis or meiosis through factors other than DNA sequence, e.g., involving histones and methylation patterns), transcription, RNA splicing, editing, translation, and turnover. RNA regulatory networks may determine most of our complex characteristics and play a significant role in disease (Mattick and Makunin, 2006). Indeed, examples of ncRNAs with specific roles in a range of developmental processes have been reported. The roles of ncRNAs are many and diverse. As they involve in the regulation of cellular differentiation and proliferation, the altered expression of key ncRNAs may lead to certain human diseases (Irminger-Finger et al., 2010). Precisely what ncRNAs do and how they work is a current hot topic in the scientific and medical fields.

MicroRNA (miRNA) is one important subcategory of ncRNAs. Most of these molecules appear to regulate target gene expression through translation repression or mRNA degradation (Cho, 2007). The biological functions of miRNAs are yet to be completely revealed. Nevertheless, increasing number of studies indicate that miRNAs are emerging as important modulators in cellular pathways and they play a key role in human diseases (Cho, 2010a). Recent reports reveal that miRNAs play significant regulatory roles in a variety of biological processes, including adipocyte differentiation, metabolic integration, insulin resistance, and appetite regulation. Investigation of these molecules and their genetic targets may potentially identify new pathways involved in complex metabolic disease processes, improving our understanding of metabolic disorders, and influence future approaches to the treatment of metabolic syndrome (Heneghan et al., 2010). Study of miRNA biogenesis and function may also add new tools for gene functional study and drug development. Indeed, the rapid discovery of many miRNA targets and their associated pathways has contributed to the development of miRNA-based therapeutics (Cho, 2010b). Emerging evidence reveals the potential of miRNA studies translating from bench to bedside applications (Cho, 2011).

Another topic of intense research interest is the small interfering RNAs (siRNAs). They inhibit gene expression by participating in RNA interference (RNAi). The identification of lipidoids facilitate high levels of specific silencing of endogenous gene transcripts when formulated with either double-stranded siRNA or single-stranded antisense 2'-O-methyl oligoribonucleotides targeting ncRNA (Akinc et al., 2008). The phosphate buffered saline-formulated locked nucleic acid-modified oligonucleotide and liposome-polycation-hyaluronic acid nanoparticle formulation modified with tumor-targeting single-chain antibody fragment enable systemic targeted delivery of siRNA (Elmén et al., 2008; Chen et al.,
2010). Over the past few years, a number of studies have revealed that siRNA is a potent method of gene silencing. RNAi has been extensively used for the fundamental research of therapeutics and has displayed great potential in clinical treatment.

The long ncRNAs (lncRNAs) are another important class of ncRNAs. In mammals and other eukaryotes, most of the genome is transcribed in a developmentally regulated manner to produce large numbers of lncRNAs (Mercer et al., 2009). Emerging evidences suggest that lncRNAs function as epigenetic regulators of transcription in human cells. Mechanistically, these lncRNAs may be involved in maintaining balanced transcription at bidirectionally transcribed loci as a method to modulate gene expression according to the selective pressures placed on the cell. The loss of this intricate bidirectional RNA-based regulatory network can result in overt epigenetic silencing of gene expression (Morris, 2009). Rather than silently accumulating in the nucleus, many lncRNAs are now believed to play important roles in nuclear architecture or in the regulation of gene expression (Chen and Carmichael, 2010).

Development of personalized medicine is closely linked to biomarkers (Villanueva et al., 2010). Since epigenetic changes often precede disease pathology, they may act as valuable diagnostic indicators for disease risk or prognostic indicators for disease progression (Kelly et al., 2010). Some aspects of epigenetics are strongly linked to ncRNAs and the role of ncRNAs as molecular biomarkers for human diseases is being supported by an increasing number of studies. Yet, there are many challenges that the researchers have to conquer before ncRNAs can be fully understood and utilized.

Firstly, it is a challenge to detect small RNAs because of their small size, lack of an open reading frame, and diverse nature (Hershberg et al., 2003). Rapid development in high-throughput platform 
technologies, such as single-nucleotide polymorphism analysis, genome-wide transcriptional profiling, microarray, BeadArray, and next generation sequencing offers the potential for revolutionary development in the study of ncRNAs. The widespread and comprehensive use of microarrays has enabled the identification of a number of ncRNAs as potential biomarkers for a variety of human diseases (Cho, 2010c).

In addition, a challenge to ncRNA studies lies in the prediction of target genes. For example, miRNAs target mRNAs that contain partially complementary sites to the miRNA. The level of complementarity is different between target site-miRNA pairs, and finding target genes has proved to be a bigger challenge than expected. Even after the identification of ncRNAs and their target genes, it is also a challenge to reveal their specific biological function and signaling pathways (Inui et al., 2010). Recent advances in bioinformatics have provided new opportunity in the molecular analysis of various human diseases in an unprecedented speed. Some studies not only reported the identified ncRNA biomarkers, but also deciphered their target genes and the underlying mechanisms. The rapid discovery of many ncRNA targets and their relevant pathways has contributed to the development of ncRNA-based therapeutics (Cho, 2010d). However, the safe and effective delivery of RNAi therapeutics in vivo remains a significant clinical challenge.

On the other hand, some particular ncRNAs can target numerous mRNAs, often in combination with other ncRNAs. Complex diseases are affected by a number of ncRNAs rather than a single ncRNA, and ncRNAs operate in highly complex regulatory networks (Kargul and Laurent, 2010). It is a challenge to identify ncRNA synergism, determine their functions at a system-wide level, and investigate disease ncRNA features in the ncRNA-ncRNA synergistic network from a holistic view (Xu et al., 2011). The development of microarrays, RT-PCR platforms, and next generation sequencing methodologies has resulted in an exponential acquisition of ncRNA profiles. However, there is no database that allows for cross-platform comparison of existing data (Farazi et al., 2011). The integration of various omics data and their functional interpretation in conjunction with clinical results may help to overcome this challenge (Cho, 2010c).

Although the biological importance of ncRNA is becoming increasingly apparent, regulation of ncRNA expression in human diseases is not fully understood. There are some questions to be addressed. ncRNA dysregulation has been linked to changes in epigenetic regulation, such as the methylation status of miRNA genes, which results in alterations in their expression levels. As some ncRNAs have an important role in self-renewal and differentiation of embryonic and tissue-specific stem cells, do they also have a regulatory role in cancer stem cells? Promising findings of a disease-associated ncRNA in one study is not adequate to support a solid report; multicenter and an independent cohort of studies would be needed to cross validate the discovery. ncRNA studies indicate their importance and potential use as theranostic tools, but the true clinical utility and the limits of their application are yet to be established. Although ncRNA-based therapeutics in several proof-of-principle experiments have demonstrated exciting effects, the developing progress of antisense or siRNA drugs has been hampered by stability, specificity, and delivery problems. Even though the LNA technology has presented a breakthrough in oligonucleotide carrier in recent years; results from clinical trials are still pending to confirm the use of this antagomir in respect to safety for human application. Nevertheless, the rapid progress in ncRNA studies points to their tremendous potential in the management of human diseases and their potential role in future therapeutics (Cho, 2009).

Non-coding RNAs is a cutting-edge topic in the scientific and medical fields, the identification of disease-related ncRNAs and their state-of-the-art treatment approaches proceed at a fast pace. Although there are many challenges to be passed through, new forms and uses of ncRNAs will continue to be discovered. However, much more work remains to be accomplished before the diverse nature of these RNA molecules can be fully understood.

\section{REFERENCES}

Akinc, A., Zumbuehl, A., Goldberg, M., Leshchiner, E. S., Busini, V., Hossain, N., Bacallado, S. A., Nguyen, D. N., Fuller, J., Alvarez, R., Borodovsky, A., Borland,
T., Constien, R., de Fougerolles, A., Dorkin, J. R., Narayanannair Jayaprakash, K., Jayaraman, M., John, M., Koteliansky, V., Manoharan, M., Nechev, L., Qin, J., Racie, T., Raitcheva, D., Rajeev, K. G., Sah, D. W., Soutschek, J., Toudjarska, I., Vornlocher, H. P., Zimmermann, T. S., Langer, R., and Anderson, D. G. (2008).A combinatorial library of lipid-like materials for delivery of RNAi therapeutics. Nat. Biotechnol. 26, 561-569.

Chen, L. L., and Carmichael, G. G. (2010). Decoding the function of nuclear long non-coding RNAs. Curr. Opin. Cell Biol. 22, 357-364.

Chen, Y., Zhu, X., Zhang, X., Liu, B., and Huang, L. (2010). Nanoparticles modified with tumor-targeting scFv deliver siRNA and miRNA for cancer therapy. Mol. Ther. 18, 1650-1656.

Cho, W.C. (2007). OncomiRs: the discovery and progress of miRNAs in cancers. Mol. Cancer 6, 60.

Cho, W. C. (2009). Role of miRNAs in lung cancer. Expert Rev. Mol. Diagn. 9, 773-776.

Cho, W.C. (2010a). MiRNAs in cancer-from research to therapy. Biochim. Biophys. Acta 1805, 209-217.

Cho, W. C. (2010b). MiRNAs as therapeutic targets for lung cancer. Expert Opin. Ther. Targets 14, 1005-1008.

Cho, W. C. (2010c). "Omics approaches in cancer research," in An Omics Perspective on Cancer Research, ed. W. C. Cho (Berlin: Springer), 1-9.

Cho, W. C. (2010d). MiRNAs: potential biomarkers for cancer diagnosis, prognosis and targets for therapy. Int. J. Biochem. Cell Biol. 42, 1273-1281.

Cho, W. C. (2011). MiRNAs in Cancer Translational Research. Berlin: Springer.

Elmén, J., Lindow, M., Schütz, S., Lawrence, M., Petri, A., Obad, S., Lindholm, M., Hedtjärn, M., Hansen, H. F., Berger, U., Gullans, S., Kearney, P., Sarnow, P., Straarup, E. M., and Kauppinen, S. (2008). LNAmediated miRNA silencing in non-human primates. Nature 452, 896-899.

Farazi, T. A., Spitzer, J. I., Morozov, P., and Tuschi, T. (2011). MiRNAs in human cancer. J. Pathol. 223, 102-115.

Heneghan, H. M., Miller, N., and Kerin, M. J. (2010). Role of miRNAs in obesity and the metabolic syndrome. Obes. Rev. 11, 354-361.

Hershberg, R., Altuvia S., and Margalit, H. (2003). A survey of small RNA-encoding genes in Escherichia coli. Nucleic Acids Res. 31, 1813-1820.

Inui, M., Martello, G., and Piccolo, S. (2010). MiRNA control of signal transduction. Nat. Rev. Mol. Cell Biol. 11, 252-263.

Irminger-Finger, I., Thomson, J. M., and Kim, J. K. (2010). MicroRNAs, a superimposed regulatory network important for development and disease. Int. J. Biochem. Cell Biol. 42, 1234-1235.

Kargul, J., and Laurent, G. J. (2010). MicroRNAs in development and disease. Int. J. Biochem. Cell Biol. 42, 1233.

Kelly, T. K., De Carvalho, D. D., and Jones, P. A. (2010). Epigenetic modifications as therapeutic targets. Nat. Biotechnol. 28, 1069-1078.

Mattick, J. S., and Makunin, I. V. (2006). Non-coding RNA. Hum. Mol. Genet. 15, R17-R29.

Mercer, T. R., Dinger, M. E., and Mattick, J.S. (2009). Long non-coding RNAs: insights into functions. Nat. Rev. Genet. 10, 155-159.

Morris, K. V. (2009). Long antisense non-coding RNAs function to direct epigenetic complexes that regulate transcription in human cells. Epigenetics 4, 296-301. 
Villanueva, A., Hoshida, Y., Toffanin, S., Lachenmayer, A., Alsinet, C., Savic, R., Cornella, H., and Llovet,J.M. (2010). New strategies in hepatocellular carcinoma: genomic prognostic markers. Clin. Cancer Res. 16, 4688-4694.

Wilusz, J. E., Sunwoo, H., and Spector, D. L. (2009). Long noncoding RNAs: functional surprises from the RNA world. Genes Dev. 23, 1494-1504.

Xu, J., Li, C. X., Li, Y. S., Lv, J. Y., Ma, Y., Shao, T. T., Xu, L. D., Wang, Y. Y., Du, L., Zhang, Y. P., Jiang, W., Li, C. Q.,
Xiao, Y., and Li, X. (2011). MiRNA-miRNA synergistic network: construction via co-regulating functional modules and disease miRNA topological features. Nucleic Acids Res. doi: 10.1093/nar/gkq832

Received: 20 December 2010; accepted: 03 January 2011; published online: 19 January 2011.

Citation: Cho WCS (2011) Grand challenges and opportunities in deciphering the role of non-coding RNAs in human diseases. Front. Gene. 2:1. doi: 10.3389/ fgene.2011.00001

This article was submitted to Frontiers in Non-Coding RNA, a specialty of Frontiers in Genetics.

Copyright $\odot 2011$ Cho. This is an open-access article subject to an exclusive license agreement between the authors and Frontiers Media SA, which permits unrestricted use, distribution, and reproduction in any medium, provided the original authors and source are credited. 